\title{
Measuring Hygiene Competence: A Vignette-Based Situational Judgement Test
}

\section{Susanne Katharina Heininger ( $\nabla$ susanne.heininger@tum.de)}

Technische Universitat Munchen Fakultat fur Medizin https://orcid.org/0000-0001-7678-8533

\section{Maria Baumgartner}

Klinikum rechts der Isar der Technischen Universitat Munchen, TUM Medical Education Center

\section{Fabian Zehner}

DIPF - Leibniz-Institut für Bildungsforschung und Bildungsinformation

\section{Rainer Burgkart}

Klinikum rechts der Isar der Technischen Universität München, Klinik und Poliklinik für Orthopädie und Sportorthopädie

Nina Söllner

Klinikum rechts der Isar der Technischen Universität München, TUM Medical Education Center

\section{Pascal O. Berberat}

Klinikum rechts der Isar der Technischen Universität München, TUM Medical Education Center

\section{Martin Gartmeier}

Klinikum rechts der Isar der Technischen Universität München, TUM Medical Education Center

\section{Research article}

Keywords: situational judgement test, hygiene, competence, assessment, item-response theory

Posted Date: May 29th, 2020

DOI: https://doi.org/10.21203/rs.3.rs-30686/v1

License: (c) (i) This work is licensed under a Creative Commons Attribution 4.0 International License. Read Full License

Version of Record: A version of this preprint was published at BMC Medical Education on July 30th, 2021. See the published version at https://doi.org/10.1186/s12909-021-02829-y. 
Measuring Hygiene Competence:

\section{A Vignette-Based Situational Judgement Test}

3

4

Susanne Heininger ${ }^{1}$, susanne.heininger@tum.de

6

Maria Baumgartner ${ }^{1}$, maria-baumgartner@arcor.de

Fabian Zehner², fabian.zehner@dipf.de

Rainer Burgkart ${ }^{3}$, rainer.burgkart@tum.de

9

Nina Söllner ${ }^{1}$, n.soellner@tum.de

Pascal O.Berberat ${ }^{1}$, berberat@tum.de

Martin Gartmeier ${ }^{1}$, martin.gartmeier@tum.de

${ }^{1}$ TUM Medical Education Center, Fakultät für Medizin, Klinikum rechts der Isar, TU München ${ }^{2}$ DIPF | Leibniz-Institut für Bildungsforschung und Bildungsinformation

Correspondence:

Dr. Susanne Katharina Heininger

Technical University of Munich

University Hospital Klinikum rechts der Isar

22

TUM Medical Education Center

23

Ismaninger Straße 22, D-81675 München

Tel. +498941409278

26

Mail: susanne.heininger@tum.de

27 


\section{A Vignette-Based Situational Judgement Test}

Abstract

\section{Background:}

Since the onset of the Corona pandemic at the beginning of 2020, the extreme importance of hygiene has once again become very clear. In the medical context, it is not easy to find suitable test formats to assess the competencies involved in "working hygienically". Pre-existing test formats usually use self-report questionnaires, which are suboptimal for this purpose.

\section{Methods:}

We designed a Situational Judgement Test (SJT) to assess hygiene competence. The SJT incorporates reliable measures and is a good predictor of job performance. The test consists of twenty pictures (items) presenting only one unambiguous hygiene lapse. Test respondents were asked (1) to note down whether they recognized a problem in the picture in respect of hygiene guidelines and (2), if yes, to describe the problem in a short verbal response. The sample comprised $n=149$ health care professionals (79.1\% female; age: $M=26.7$ years, $S D=7.3$ years) working as clinicians or nurses. The written responses were rated by two independent raters with high agreement $(\alpha>.80)$, indicating high reliability of the measurement. We then used Item Response Theory (IRT) for data analysis.

\section{Results:}

The reported IRT analyses show that the test is suitable to assess hygiene competence and that it is possible to distinguish between persons demonstrating different levels of ability for seventeen of the twenty items/pictures), expecially for the range of low to medium person abilities. 
52

Conclusions:

53

The test in its present form can be used to assess the hygiene competence of medical students,

54 medical doctors, nurses and trainee nurses in cross-sectional measurements. In order to broaden the

55 difficulty spectrum of the current test, additional test items with higher difficulty should be

56 developed. The Situational Judgement Test designed to assess hygiene competence can be helpful in

57 testing and teaching the ability of working hygienically.

58

59

60

61

62

63

64

Keywords: situational judgement test; hygiene; competence; assessment; item-response theory;

65

66

67 
Nosocomial infections are a serious challenge in modern patient care [1] and have recently been subject of intense research [2]. In order to prevent hospital-acquired infections (HAI) and improve patient and staff safety, hygiene is essential. In pandemics like COVID-19 in the year 2020, the prevention of infection through good hygiene is crucial. Some pre-existing forms of intervention are designed to increase awareness and improve attitudes towards hygiene in daily clinical work [3-5]. While there are numerous single studies (e.g. $[6],[7,8]$ and a number of meta-analyses $[9,10]$ on the issue of hygiene, these mostly focus on interventions for improving hand hygiene. A massive international campaign for the prevention of infection, the "My Five Moments of Hand Hygiene"program [11], also puts the emphasis on hand hygiene. We argue, however, that the focus on hand hygiene is rather narrow because health care staff draw upon a more differentiated repertoire of cognitive resources in order to adhere to hygiene standards in daily work. This argument is supported by the WHO Guidelines on Core Components of Infection Prevention and Control (IPC) Programmes [12] . These guidelines, based on the WHO Core Components for Infection Prevention and Control Report, were extended in 2016, highlighting additional areas for preventing infections in health care facilities worldwide, especially in acute health care facilities: personnel and facility resources, including workload, staffing, materials and equipment (guideline recommendations numbers seven and eight). This points up the need to develop a more differentiated concept of hygiene competence - which also requires more sophisticated measurement strategies.

In a recent article, Gartmeier et al. [13] proposed a multidimensional model of hygiene competence consisting of three dimensions, knowledge, skills and attitudes. They argue that knowledge about why hygiene is important and how it can be maintained in specific clinical situations is necessary, but not sufficient. Moreover, health care staff need specific skills in order to apply their knowledge in hygienic patient care. Finally, specific attitudes are required, e.g. maintaining hygienic working procedures despite extreme time pressures and understaffing. What this model proposes (especially compared to more general models such as the "My Five Moments of Hand Hygiene" [11]) is that in 
order to uphold hygiene across a multitude of particular health care tasks (e.g., providing artificial respiration or replacing a patient's bladder catheter), specific procedures are performed which require synergy between all three dimensions of hygiene [13] described above.

In order to improve hygiene competence more effectively in the future, we argue that psychometrically promising instruments should be designed to assess this competence. The approach to measurement should take account of the fact that health care staff manage to synthesize different resources - knowledge, skills and attitudes - in order to maintain hygiene in their daily work practice. We propose that a Situational Judgement Test (SJT) requiring test respondents to identify the presence or absence of hygiene problems in images of clinical situations is a promising approach in this respect. We elaborate this conjecture in what follows.

\section{Assessment of hygiene competence}

Currently, researchers primarily use self-report questionnaires to assess hygiene-related constructs with regard to attitudes and practices (see e.g. [6] [14]). Evidence shows, however, that such instruments are suboptimal in measuring competencies [15], partly because health care staff systematically overestimate the number of hygiene-related behaviors they perform in clinical practice [16]. More objective measures should therefore be developed to assess hygiene competence. In order to measure competencies, direct observations or simulations are optimal but are resource-intensive and cannot be easily standardized $[17,18]$. With regard to hand hygiene, different observational tools are available [19-21], but no current instrument adopts the more comprehensive measurement approach suggested by the above mentioned model of hygiene competence [13]. Drawing upon this model, we propose that a SJT is a promising, time- and costefficient method of assessment. SJTs require respondents to make knowledge-based judgements of scenarios displayed as short texts, pictures or videos [22] [23]. Properly designed SJTs yield reliable measures, they are good predictors of job performance and are well accepted by test respondents [23]. Moreover, SJTs allow assessments to be conducted in a standardized way - which is less of a 
drain on scarce resources and time $[22,23]$. Such instruments are hence increasingly used in the medical context, for instance, in context of medical admission procedures [22-24].

In the Methods section below, we describe our design of an SJT intended to measure hygiene competence, or, more specifically, the dimensions knowledge and practices/skills. This test requires respondents to make knowledge-based judgements of still-images of common clinical work procedures and situations. Respondents have to apply their knowledge in order to judge whether the clinical practices shown in the images are carried out in compliance with hygiene standards. This assessment strategy relates to the second level in Miller's widely used prismatic model of clinical competence [17], which represents "knows how" as the (cognitive) application of knowledge. We will report a pilot study with an initial version of this SJT, focusing upon two research questions: (RQ1) Does the SJT comply with competence test quality requirements described in context of the ItemResponse-Theory (IRT)? (RQ2) Is it possible to cover a broad spectrum of item difficulty / person ability regarding hygiene competence by means of the SJT?

\section{SJT design and measurement strategy}

Our SJT is designed to assess hygiene competence and consists of twenty picture vignettes (see Figure 1 for a sample item). Each vignette shows at least one health care provider (nurse $[\mathrm{f} / \mathrm{m}]$ or clinician) and a patient (where appropriate) in a clinical situation in which hygiene is a relevant issue. The test items were constructed in the following way: Every item shows only one unambiguous fault (two items show no fault). The situations displayed (medical equipment and rooms) are authentic reconstructions of clinical situations taken in different rooms (OR, patient room, treatment room, intensive care room) of the Medical Training Center (MTC) of the München rechts der Isar (MRI) university hospital. All situations shown in the test items focus on different actions of clinicians and nurses ( $f / m$; see Table 1 ). The reason for the interdisciplinary setup lies in the fact that hygiene 
competence often needs to be applied in collaborative settings. Hygiene fails when two professions fail to achieve inter-professional alignment. A thorough knowledge of hygiene is therefore important regardless of profession. As described above, these items refer to the hygiene model dimensions knowledge and skills [13]. The attitudes-dimension of the competence model could not directly be represented in the test. All persons depicted were non-professional actors from whom full consent was obtained. Materials, rooms, equipment and procedures were pictured as realistically as possible (e.g. picture vignettes showing a situation in OR were taken in a simulation OR with original materials). All situations displayed were conceptualized in close cooperation with and reviewed by nurses and medical doctors with extensive clinical experience to ensure the most realistic portrayal of situations in the pictures.

\section{Insert Figure 1 about here}

The generated picture vignettes were compiled into a paper-pencil questionnaire. For each vignette, respondents were asked (1) to note down whether they recognize a problem in the picture regarding hygiene guidelines (dichotomous: yes/no) and (2), if yes, to describe the problem in a short verbal response. For each item, an ideal solution was developed based on scientific literature and national clinical hygiene guidelines. All picture vignettes and solutions were reviewed in cooperation with the group of local hygiene experts (who were advanced clinicians, nurses [f/m] and specialists from the local hygiene department). Only completely correct answers were credited if (1) the respondent recognized an incorrect (unhygienic) action in the picture and (2) described the hygiene lapse correctly (matching to the model solution). In contrast, respondents were not credited if they just recognized the incorrect (unhygienic) action, but could not point out what exactly was wrong (e.g. sterile gloves missing). In the present study, three raters (two pairs: raters $A+B$, raters $B+C$ ) evaluated all given answers and took a pass-fail-decision (dichotomous) based on the model solution. Krippendorff's alpha [25] was calculated to measure the inter-rater reliability of the rating procedure. 


\begin{tabular}{|c|c|c|}
\hline Item & Content / Issue & Hygiene problem \\
\hline 1 & Incorrect wearing of surgical face mask & yes \\
\hline 2 & Incorrect hand posture during pre-surgery dressing & yes \\
\hline 3 & Nurse incorrectly standing next to a sterile table & yes \\
\hline 4 & Wearing of personal items (wristwatch) at bedside & yes \\
\hline 5 & $\begin{array}{l}\text { Personal sluice (incorrect changing of personal to work } \\
\text { clothing) }\end{array}$ & Yes \\
\hline 6 & Non-sterile suctioning of mucus & Yes \\
\hline 7 & Putting blood sampling set down on patient's bedside & Yes \\
\hline 8 & Personal hygiene - nurse's hair touching patients bed & Yes \\
\hline 9 & $\begin{array}{l}\text { Sterile hand gloves being transported in nurse's dress } \\
\text { pocket }\end{array}$ & Yes \\
\hline 10 & Personal hygiene - tie touching patient's bed blanket & Yes \\
\hline 11 & Personal hygiene (artificial fingernails) in clinical practice & Yes \\
\hline 12 & $\begin{array}{l}\text { Reaching into care trolley drawer with contaminated hand } \\
\text { gloves }\end{array}$ & Yes \\
\hline 13 & Using care trolley in isolated patient room & Yes \\
\hline 14 & Wearing medical work clothes in public areas & Yes \\
\hline 15 & No-touch-technique & No \\
\hline 16 & Hygienically transporting medical file on patient bed & No \\
\hline 17 & Typing on computer keyboard wearing sterile hand gloves & Yes \\
\hline 18 & Carrying infusion bottle in nurse's dress pocket & Yes \\
\hline 19 & Opening sterile syringe packaging & Yes \\
\hline 20 & Wearing personal items (jewelry) in clinical practice & Yes \\
\hline
\end{tabular}



years) working as clinicians or nurses (f/m; see Table 2 for overview).

Table 2. Sample professions and professional experiences

\begin{tabular}{|c|c|c|c|}
\hline \multirow[t]{2}{*}{ Profession } & \multirow{2}{*}{$\begin{array}{c}\text { Frequency } \\
\text { in } \%\end{array}$} & \multicolumn{2}{|c|}{ Professional Experience in Years } \\
\hline & & $M(S D)$ & Freq. in $\%$ \\
\hline Medical Student & 40.8 & $5.6(.85)$ & \\
\hline Medical Doctor & 4.8 & $7.0(8.49)$ & \\
\hline \multirow[t]{2}{*}{ Trainee Nurse $^{a}(\mathrm{f} / \mathrm{m})$} & 34.7 & $2.2(1.00)$ & First year: 41.7 \\
\hline & & & Third year: 58.3 \\
\hline Nurse (f/m) & 19.0 & $14.3(13.81)$ & \\
\hline $\begin{array}{l}\text { Trainee operating room } \\
\text { technician (ORT) }\end{array}$ & 0.7 & $1.0(0)$ & First year: 100 \\
\hline \multicolumn{4}{|c|}{$\begin{array}{l}\text { a In Germany, nursing education is a vocational college training, which is not rooted at a university. Trainee nurses spent } \\
\text { three years at a vocational school for theoretical and practical education }(2,100 \mathrm{~h}) \text {. Additionally, trainee nurses serve } 2,500 \\
\text { hrs at a teaching hospital for practical training. } \\
\text { b In Germany, ORT education is based at a vocational college as well. The theoretical education covers } 1,600 \mathrm{hrs,} \mathrm{the} \\
\text { practical training includes } 3,000 \mathrm{hrs} \text { at a teaching hospital. }\end{array}$} \\
\hline
\end{tabular}

Analysis

We used Item Response Theory (IRT) for data analysis. The IRT framework provides a family of probabilistic models, which allow for simultaneous estimation of respondent ability and item difficulty [26]. This way, one can model "the interaction between an individual item and an individual examinee" [27] by using the Rasch Model, the most restrictive IRT model with the best measurement properties. Only if a test has been shown to produce data conforming to the Rasch Model is it fair to use the sum score. This model can be used to estimate unidimensional latent abilities [26]. The model assumes that the probability $P\left(X_{v, i}\right)$ of a person $v$ giving a certain answer $X$ for an item $i$ depends on two factors: (1) the item difficulty, $\sigma_{i}$, and (2) the person's ability $\theta_{v}[28]$, which are estimated simultaneously on the same scale. This means, the numbers for person abilities and item 
difficulties can be directly compared. If a person with ability $\theta_{v}=1.1$ responds to an item with

192 difficulty $\sigma_{i}=1.1$, the probability of a correct response is 50 percent. But if the difference between

193 ability and difficulty is largely positive or negative, the probability of a correct response gets close to

Table 3. Krippendorff's alpha for rater sets

\begin{tabular}{lc}
\hline Rater Group & Krippendorff's alpha \\
\hline Raters A+B & .81 \\
Raters B+C & .86 \\
\hline
\end{tabular}

100 percent or 0 percent, respectively. Negative parameter values indicate simple items or low ability; positive values refer to difficult items or high ability [29]. Mathematically, these relationships are modelled by the following equation:

$$
P X i,=1 \sigma i, \theta v=e(\theta v-\sigma i) 1+e(\theta v-\sigma i)
$$

In order to test whether collected data are compliant with the Rasch Model, it is crucial to confirm the model's assumption of specific objectivity [26]. This means that different subpopulations in a sample should not differ significantly regarding item difficulties. To measure this, is current state-ofthe-art involves splitting a sample into subsamples and comparing the resulting item difficulties using the Andersen Likelihood Ratio Test [30]. Since the null hypothesis assumes no differences, it is common to use three split criteria, and to apply a Type-I-risk of $\alpha=.01$, so this risk inflates to no more than $\alpha=.05$ across all comparisons [26]. In this study, we used the split criteria raw score median (comparing two achievement groups), gender and age median. IRT analyses were carried out using the R packages $e R m$ and $P P$ [31-33].

Inter-rater-reliability

We calculated Krippendorff's alpha for inter-rater reliablity and found a strong agreement $(\alpha \geq .80$;

see Table 3).

Note. Rater A: student rater; rater $\mathrm{B} / \mathrm{C}$ : research associates 
Based on Andersen's Likelihood Ratio Test, three of the twenty items had to be excluded in order to reach sufficient model fit. Using the remaining seventeen items, Rasch Model conformity could be assumed for the instrument - see test statistics displayed in Table 4. Fisher's $z$-Test indicated which item had to be excluded. Figure 1 shows the resulting item difficulty parameters and person a person's ability.

Table 4. Rasch Model Conformity Achieved for the Final Scale with 17 Items

\begin{tabular}{rcccc}
\hline Split Criterion & LRT $\chi^{2}$ & $\mathrm{df}$ & $\chi^{2} \mathrm{df}, \alpha=.01$ & $p$ \\
\hline High / Low Achievement & 28.48 & 16 & 32.00 & .028 \\
Female / Male & 31.81 & 16 & 32.00 & .011 \\
Younger / Older & 31.87 & 16 & 32.00 & .010 \\
\hline
\end{tabular}

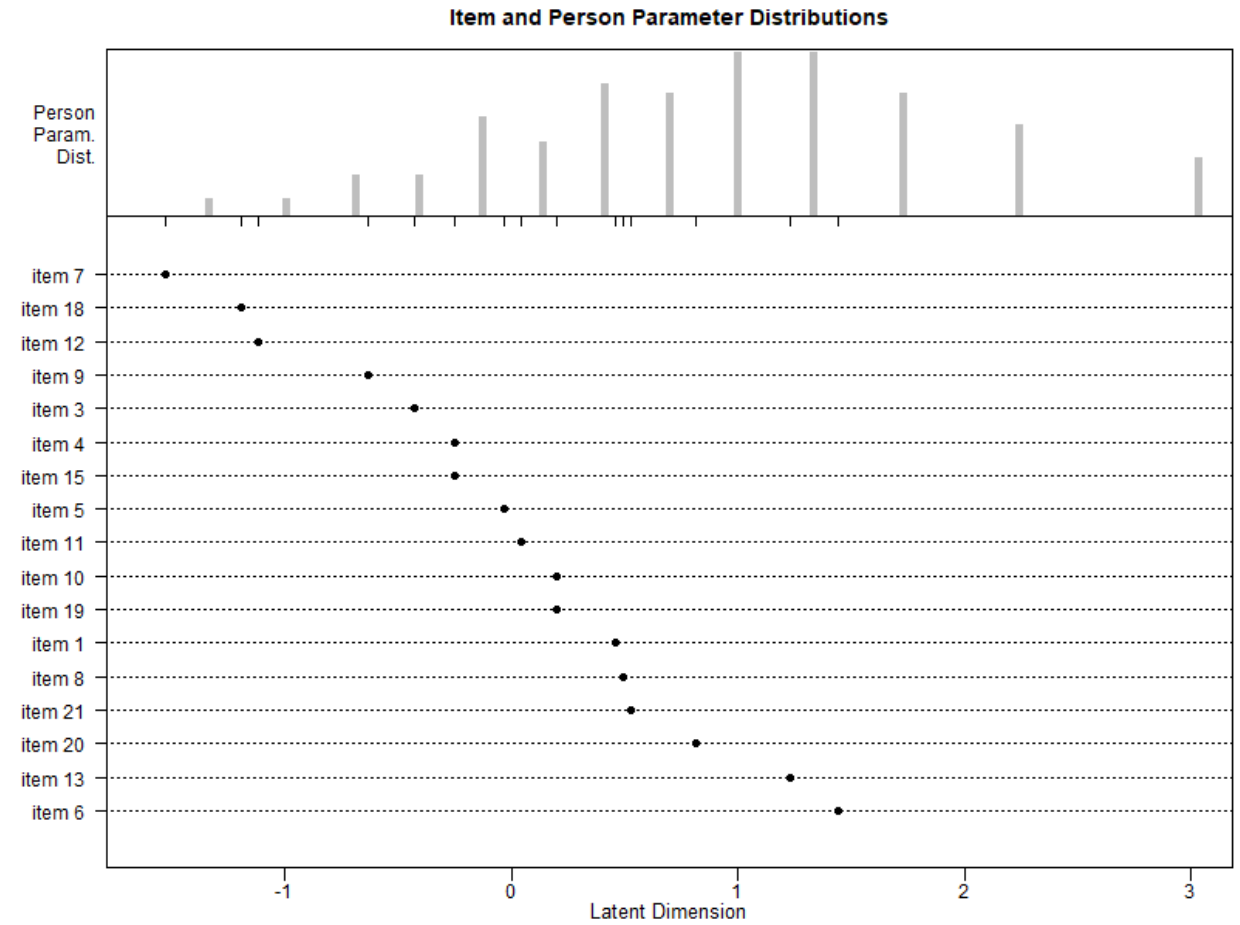

Figure 1. Person ability (top) and item (bottom) parameter distribution for the final instrument 
As is apparent, our test items cover a difficulty spectrum from low to medium; no item showed a high degree of difficulty. Some test respondents, however, showed higher ability, meaning they were seemingly able to solve all test items (cf. upper section of Figure 1).

\section{Discussion}

In this paper, we have described a novel, time and cost-efficient, yet psychometrically promising method of assessing hygiene competence based upon a recent theoretical model [13]. The developed SJT, originally consisting of twenty picture items, offers a feasible way of assessing hygiene competence. The responses given by the study participants were rated by two independent raters with high agreement $(\alpha>.80)$, indicating the high reliability of the measurement. Further, the reported IRT analyses show that the test is suitable to assess hygiene competence and that it is possible to distinguish between persons with different levels of ability. After exclusion of three items, the remaining seventeen items showed a good measurement quality (RQ1). Further, the results showed that our test items cover the range of low to medium person ability regarding hygiene competence. The current results show that the initial test version does not contain items with sufficient difficulty to discriminate for very high abilities (i.e., highly able test respondents would answer all items correctly, RQ2). Nevertheless, because we did not observe a strong ceiling effect, the test in its present form can be used to assess hygiene competence of medical students, medical doctors, nurses and trainee nurses in cross-sectional measurements.

A general conclusion to be drawn from the present results is that the described measurement strategy offers a promising means of estimating professionals' hygiene competence. Nevertheless, some critical remarks have to be made regarding the test in its current version: First, the test covers a broad range of clinical situations, some of which may be regarded as special cases (e.g. items 1, 2, 3 and 5 showing situations in the OR). For this reason, it could be argued that these items are very difficult to answer for respondents who have no or only very limited experience regarding this very 
specific area of health care. However, as is apparent from Table 1, the operating-room items 1, 3 and 5 all had medium difficulty levels. Thus, these outcomes do not suggest that respondents had substantial difficulties in answering these items because they were not familiar with these situations.

In order to further develop the current instrument, several perspectives are recommended: First, we suggest a data-based strategy to determine what is measured by the current instrument. To achieve this, correlations with data collected with existing hygiene-related measures (e.g. [6] [14]) should be examined. In addition, correlations with performance in simulated situations or with observations in clinical practice would be useful. In order to broaden the difficulty spectrum of the current test, additional test items with higher difficulty could be developed. This can be achieved in terms of content by using more specific situations, e.g. in the OR, or with special treatment situations. An alternative solution would be to use a more complex item format, such as short videos instead of picture vignettes. Hygiene lapses would be viewable only for a short interval, making them more authentic and closer to real-world performance. The video format would provide an approach more consistent with reality, but would also require investigating the attention processes of the test respondents. Furthermore, the video format can be seen as a connection between a static photo and the fast-moving reality of real-world health care scenarios. It would allow complex reality to be presented with the options of stopping or slowing the playback. Video items could be used in testing hygiene competence as well as in teaching hygiene competence.

\section{Conclusions}

Since the onset of the Corona pandemic at the beginning of 2020, the extreme importance of hygiene is clear to everyone in the health care sector and beyond. Teaching hygiene to medical students may thus receive even greater attention than before. For teaching purposes, it is not always easy to find suitable test formats that make it possible to test newly acquired skills. It is therefore even more important to be able to test the results of the competencies taught. This Situational 
276 Judgment Test, which is designed to assess hygiene competence, may help to address the urgent

277 need to teach and test the ability to work hygienically in the medical context.

\section{Abbreviations}

COVID-19 Coronavirus disease (SARS-CoV-2)

$281 \quad$ IPC

Infection prevention and control. Infection prevention and control (IPC) of WHO is a scientific approach and practical solution designed to prevent harm caused by infection to patients and health workers.

Item Response Theory

University hospital rechts der Isar (located to the right of the Isar river)

Medical training center

OR

Operating room

Research question

Situational judgement test

\section{Declarations}

295 All procedures performed in studies involving human participants were in accordance with the

296 ethical standards of the institutional and/or national research committee and with the 1964 Helsinki declaration and its later amendments or comparable ethical standards. The data acquisition 
299 rechts der Isar der Technischen Universität, München).

\section{Availability of data and materials}

All data and materials are available from the manuscript or from the corresponding author upon request.

The authors declare that they have no competing interests.

\section{Funding}

Not applicable.

\section{Authors' contributions}

310 All authors have contributed sufficiently to the project to be included as authors. MG POB RB MB and

311 SH designed the study. MG POB NS RB MB and SH coordinated the study and the data acquisition. FZ MG NS MB and SH performed the statistical analyses. SH MG FZ POB drafted the manuscript. All authors revised the manuscript for important intellectual content. All authors read and approved the

314 final manuscript.

\section{Acknowledgements}

316 Many thanks to all medical students who participated in this study and to the many voluntary

317 supporters. No internal or external sources of funding were used to support this work. 
DIPF | Leibniz-Institut für Bildungsforschung und Bildungsinformation

322

323

324

325

326

327

328

329

330

331

332

333

334

335

336

337

338

339

340

341

342

343

344

345

346

347

348

349

350

351

352

353

354

Fabian Zehner

Klinik und Poliklinik für Orthopädie und Sportorthopädie, Fakultät für Medizin, Klinikum rechts der Isar, TU München

Rainer Burgkart

\section{References}

1. Khan HA, Baig FK, Mehboob R. Nosocomial infections: Epidemiology, prevention, control and surveillance. Asian Pacific Journal of Tropical Biomedicine. 2017;7:478-82. doi:10.1016/j.apjtb.2017.01.019.

2. World Health Organization. WHO Guidelines on Hand Hygiene in Health Care: First Global Patient Safety Challenge. Clean Care is Safer Care. Geneva: WORLD HEALTH ORGANIZATION; 2009.

3. Cresswell P, Monrouxe LV. 'And you'll suddenly realise 'I've not washed my hands': medical students', junior doctors' and medical educators' narratives of hygiene behaviours. BMJ Open. 2018;8:e018156. doi:10.1136/bmjopen-2017-018156.

4. Gilmartin H, Saint S, Rogers M, Winter S, Snyder A, Quinn M, Chopra V. Pilot randomised controlled trial to improve hand hygiene through mindful moments. BMJ Qual Saf. 2018;27:799806. doi:10.1136/bmjqs-2017-007359.

5. Pittet D, Hugonnet S, Harbarth S, Mourouga P, Sauvan V, Touveneau S, Perneger TV. Effectiveness of a hospital-wide programme to improve compliance with hand hygiene.: Infection Control Programme. Lancet. 2000;356:1307-12.

6. Graf K, Chaberny IF, Vonberg R-P. Beliefs about hand hygiene: a survey in medical students in their first clinical year. Am J Infect Control. 2011;39:885-8. doi:10.1016/j.ajic.2010.08.025.

7. Fichtner A, Haupt E, Karwath T, Wullenk K, Pöhlmann C, Jatzwauk L. A single standardized practical training for surgical scrubbing according to EN1500: effect quantification, value of the standardized method and comparison with clinical reference groups. GMS Z Med Ausbild. 2013;30:Doc24. doi:10.3205/zma000867.

8. Fuller C, Michie S, Savage J, McAteer J, Besser S, Charlett A, et al. The Feedback Intervention Trial (FIT)--improving hand-hygiene compliance in UK healthcare workers: a stepped wedge cluster randomised controlled trial. PLOS ONE. 2012;7:e41617. doi:10.1371/journal.pone.0041617.

9. Erasmus V, Daha TJ, Brug H, Richardus JH, Behrendt MD, Vos MC, van Beeck EF. Systematic review of studies on compliance with hand hygiene guidelines in hospital care. Infect Control Hosp Epidemiol. 2010;31:283-94. doi:10.1086/650451.

10. Kingston L, O'Connell NH, Dunne CP. Hand hygiene-related clinical trials reported since 2010: a systematic review. J Hosp Infect. 2016;92:309-20. doi:10.1016/j.jhin.2015.11.012. 
11. Sax H, Allegranzi B, Uçkay I, Larson E, Boyce J, Pittet D. 'My five moments for hand hygiene': a user-centred design approach to understand, train, monitor and report hand hygiene. J Hosp Infect. 2007;67:9-21. doi:10.1016/j.jhin.2007.06.004.

12. World Health Organization. Guidelines on core components of infection prevention and control programmes at the national and acute health care facility level. WORLD HEALTH ORGANIZATION; 2016.

13. Gartmeier M, Baumgartner M, Burgkart R, Heininger SK, Berberat PO. Why Hand Hygiene is Not Sufficient: Modeling Hygiene Competence of Clinical Staff as a Basis for its Development and Assessment. GMS Journal Med Ed. 2019;36.

14. Richter A, Chaberny IF, Surikow A, Schock B. Hygiene in medical education - Increasing patient safety through the implementation of practical training in infection prevention. GMS Journal Med Ed. 2019;36:1-16. doi:10.3205/zma001223.

15. Hartig J, Klieme E, Leutner D, editors. Assessment of Competencies in Educational Contexts. 1st ed. Göttingen: Hogrefe Publishing; 2008.

16. Jenner EA, Fletcher BC, Watson P, Jones FA, Miller L, Scott GM. Discrepancy between selfreported and observed hand hygiene behaviour in healthcare professionals. J Hosp Infect. 2006;63:418-22. doi:10.1016/j.jhin.2006.03.012.

17. Mehay R, Burns R. Miller's Pyramid of Clinical Competence. In: Mehay R, editor. The essential handbook for GP training and education. London: Radcliffe Publishing Ltd; 2012. p. 414.

18. Heininger SK. Die Qualität bildungswissenschaftlicher Evidenz erkennen. Wiesbaden: Springer Fachmedien Wiesbaden; 2019.

19. McAteer J, Stone S, Fuller C, Charlett A, Cookson B, Slade R, et al. Development of an observational measure of healthcare worker hand-hygiene behaviour: the hand-hygiene observation tool (HHOT). Journal of Hospital Infection. 2008;68:222-9. doi:10.1016/j.jhin.2007.12.009.

20. McCalla S, Reilly M, Thomas R, McSpedon-Rai D. An automated hand hygiene compliance system is associated with improved monitoring of hand hygiene. Am J Infect Control. 2017;45:492-7. doi:10.1016/j.ajic.2016.12.015.

21. Sax H, Allegranzi B, Chraïti M-N, Boyce J, Larson E, Pittet D. The World Health Organization hand hygiene observation method. Am J Infect Control. 2009;37:827-34. doi:10.1016/j.ajic.2009.07.003.

22. Whetzel DL, McDaniel MA. Situational judgment tests: An overview of current research. Human Resource Management Review. 2009;19:188-202. doi:10.1016/j.hrmr.2009.03.007.

23. Patterson F, Zibarras L, Ashworth V. Situational judgement tests in medical education and training: Research, theory and practice: AMEE Guide No. 100. Med Teach. 2016;38:3-17. doi:10.3109/0142159X.2015.1072619.

24. Kiessling C, Bauer J, Gartmeier M, Iblher P, Karsten G, Kiesewetter J, et al. Development and validation of a computer-based situational judgement test to assess medical students' communication skills in the field of shared decision making. Patient Educ Couns. 2016;99:185864. doi:10.1016/j.pec.2016.06.006. 
25. Hayes AF, Krippendorff K. Answering the call for a standard reliability measure for coding data. Communication Methods and Measures. 2007;1:77-89.

26. Kubinger KD. Psychological Test Calibration Using the Rasch Model-Some Critical Suggestions on Traditional Approaches. International Journal of Testing. 2005;5:377-94. doi:10.1207/s15327574ijt0504_3.

27. van der Linden WJ, editor. Handbook of modern item response theory: Volume three: Applications. New York: Springer; 2010.

28. Geiser C, Eid M. Item-Response-Theorie. In: Wolf C, Best H, editors. Handbuch der sozialwissenschaftlichen Datenanalyse. 1st ed. Wiesbaden: VS Verlag für Sozialwissenschaften / Springer Fachmedien Wiesbaden GmbH Wiesbaden; 2010. p. 305-327. doi:10.1007/978-3-53192038-2_14.

29. Bühner M. Einführung in die Test- und Fragebogenkonstruktion. 3rd ed. München: Pearson Studium; 2011.

30. Andersen EB. A goodness of fit test for the rasch model. Psychometrika. 1973;38:123-40. doi:10.1007/BF02291180.

31. Reif M. PP: Estimation of person parameters for the 1,2,3,4-PL model and the GPCM. R package version 0.6.1. 2017. https://github.com/manuelreif/PP.

32. Mair P, Hatzinger R, Maier M. J. eRm: Extended Rasch Modeling. 0.16-2. 2018. http://erm.rforge.r-project.org/.

33. R Core Team. R: A language and environment for statistical computing. https://www.Rproject.org/. 